Bull. Austral. Math. Soc.

20D10, 20D15

VOL. 69 (2004) [25-33]

\title{
SATURATED FORMATIONS AND SYLOW NORMALISERS
}

\author{
A. D'Aniello, C. De Vivo and G. Giordano
}

Sufficient conditions are provided in order that some classes of finite soluble groups, defined by properties of the Sylow normalisers, are saturated formations.

\section{0 . INTRODUCTION}

Let $h: \mathbb{P} \rightarrow$ \{group classes be a function which associates with each $p$ a (possibly empty) class of groups $h(p)$, contained in some universe $\mathcal{B}$ of finite groups. The operation $\mathrm{N}$ on the functions $\mathbb{P} \rightarrow$ \{group classes $\}$ is defined as follows:

$$
\mathrm{N} h:=\left(G \in \mathcal{B} \mid \mathrm{N}_{G}\left(G_{p}\right) \in h(p), \text { for every prime } p \text { which divides }|G|\right)
$$

where $G_{p} \in \operatorname{Syl}_{p}(G)$.

In this paper we provide sufficient conditions in order that $\mathrm{N} h$ is a saturated formation, we suggest a way to construct a wide class of such saturated formations and a local definition for them.

It is easy to observe that, if $h(p)$ is Q-closed (closed under epimorphic images), for every prime $p$, then $\mathrm{N} h$ is $\mathrm{Q}$-closed, whereas nothing analogous occurs for other frequently used closure operations. For instance, if $h(p)$ is the class $\mathcal{T}$ of finite groups with ordered Sylow tower (for every prime $p$ ), $\mathcal{T}$ is an S-closed saturated Fitting formation, instead $\mathrm{N} \mathcal{T}=\mathrm{N} h$ is neither a formation nor a Fitting class ( $\mathrm{N} \mathcal{T}$ is closed under none of the operations $\left.R_{o}, N_{0}, S_{n}\right)$. The classes $N h$ can have some interesting properties, though they do not inherit the closure properties of the classes $h(p)$, an example is provided by the class $\mathrm{N} \mathcal{U}$, where $\mathcal{U}$ is the formation of supersoluble groups. The class $N \mathcal{U}$ has been studied in 1988 by Fedri and Serena [5] and in 1991 by the same authors with Bryce [3].

The operation $\mathrm{N}$ was introduced in 1970 by Glaubermann [6], who proved that, if $x$ is the formation function defined for each $p \in \mathbb{P}$ by $x(p)=\mathcal{S}_{p}=$ class of $p$-groups, then $\mathrm{N} x=\bigcup_{p \in \mathbb{P}} \mathcal{S}_{p}$.

In 1986 Bianchi, Gillio and Hauck [2], generalising the cited result of Glaubermann, proved that $\mathrm{N} \mathcal{N}=\mathcal{N}$, where $\mathcal{N}$ is the formation of nilpotent groups. A further generalisation was obtained in 1999 by Ballester-Bolinches and Shemetkov [1], who proved

Received 15th April, 2003

Copyright Clearance Centre, Inc. Serial-fee code: 0004-9727/04 \$A2.00+0.00. 
that, if $y$ is the formation function which associates with each prime $p$ the formation $y(p)=\mathcal{F}_{p^{\prime}} \mathcal{S}_{p}$ of $p$-nilpotent groups, then $\mathrm{N} y=\mathcal{N}$

As a concrete example we shall consider a saturated formation function introduced by Huppert in [7], and as corollaries we get the results of Fedri and Serena on the class $\mathrm{N} \mathcal{U}$ and, for soluble groups only, the result of Ballester-Bolinches and Shemetkov [1]. An interesting corollary is also the following one:

The class of soluble groups, in which normalisers of Sylow p-subgroups are p-supersoluble, is a saturated formation (see Theorem B).

Most of our notation is standard and can be found in [4]. "Group" will stand for "finite soluble group".

\section{The main Result}

In this section we prove the following theorem:

THEOREM 1.1. Let $g$ be a formation function and $\pi$ a set of primes such that $\mathcal{S}_{p^{\prime}} \cap \mathcal{S}_{\pi^{\prime}} \subseteq g(p) \subseteq \mathcal{S}_{\pi^{\prime}}$, for all primes $p$. Then $\mathrm{N} g$ is a formation. Moreover, if $g$ is a saturated formation function, then $\mathrm{N} g$ is a saturated formation.

ProOF: We have already observed that $\mathrm{N} g$ is a homomorph. Now we prove that $\mathrm{N} g$ is $\mathrm{R}_{0}$-closed. On the assumption that it is not let $G$ be a group in $\mathrm{R}_{0}(\mathrm{~N} g) \backslash \mathrm{N} g$. Since $G$ is in $\mathrm{R}_{0}(\mathrm{~N} g)$, it has two normal subgroups $K_{1}$ and $K_{2}$ such that $G / K_{i} \in \mathrm{N} g$ $(i=1,2)$, and $K_{1} \cap K_{2}=1$.

On the other hand, since $G \notin \mathrm{N} g$, there exists a prime $p$ dividing $|G|$ such that $N_{G}\left(G_{p}\right) \notin g(p)$. If $p$ divides $G / K_{i}$ (for $i=1,2$ ), we obtain

$$
N_{G}\left(G_{p}\right) / K_{i} \cap N_{G}\left(G_{p}\right) \in g(p)
$$

from which the contradiction $N_{G}\left(G_{p}\right) \in g(p)$ follows, because $g(p)$ is $\mathbf{R}_{0}$-closed. Suppose, without loss of generality, $G / K_{1} \in \mathcal{S}_{p^{\prime}}$. Since $K_{1} \cap K_{2}=1$, we get $N_{G}\left(G_{p}\right) / K_{2} \cap N_{G}\left(G_{p}\right) \in g(p)$. If $G / K_{1} \in \mathcal{S}_{\pi^{\prime}}$, we have $N_{G}\left(G_{p}\right) / K_{1} \cap N_{G}\left(G_{p}\right)$ $\in \mathcal{S}_{p^{\prime}} \cap \mathcal{S}_{\pi^{\prime}} \subseteq g(p)$ and so once more we have the contradiction $N_{G}\left(G_{p}\right) \in g(p)$. Let $q \in \pi$ be a prime dividing $\left|G / K_{1}\right|$. Since $G / K_{1} \in \mathrm{N} g$, we have $N_{G}\left(G_{q}\right) / K_{1} \cap N_{G}\left(G_{q}\right)$ $\in g(q) \subseteq \mathcal{S}_{\pi^{\prime}}$; it follows, as $q \notin \pi^{\prime}$, that $q$ does not divide $\left|N_{G}\left(G_{q}\right) / K_{1} \cap N_{G}\left(G_{q}\right)\right|$ and therefore $G_{q} \subseteq K_{1}$, obtaining the contradiction that $q$ does not divide $\left|G / K_{1}\right|$. Hence the assumption that $\mathrm{N} g$ is not $\mathrm{R}_{0}$-closed is false.

Now we are going to prove that, if $g(p)$ is saturated for every prime $p$, then $\mathrm{N} g$ is saturated. If not then let $G$ be a group of minimal order in $E_{\Phi}(\mathrm{N} g) \backslash \mathrm{N} g$. A routine argument shows that $G$ is monolithic and, if $N$ is the socle of $G$, we have, for some prime $q, N \subseteq \Phi(G) \subset O_{q}(G)=$ Fit $(G)$ and $G / N \in \mathrm{N} g$. On the other hand, since $G \notin \mathrm{N} g$, there exists a prime $p$ dividing $|G|$ such that $N_{G}\left(G_{p}\right) \notin g(p)$. Now, since 
$G / N \in \mathrm{N} g$ and $q$ divides $|G / N|$ (because $N \subseteq \Phi(G)$ ), we have $N_{G}\left(G_{q}\right) / N \in g(q)$ $\subseteq \mathcal{S}_{\pi^{\prime}}$ and $N_{G}\left(G_{p}\right) / N \cap N_{G}\left(G_{p}\right) \in g(p) \subseteq \mathcal{S}_{\pi^{\prime}} ;$ consequentely $p, q \notin \pi$ and therefore $N_{G}\left(G_{p}\right) / G_{p} \in \mathcal{S}_{p^{\prime}} \cap \mathcal{S}_{\pi^{\prime}} \subseteq g(p)$.

If $q \neq p$, we get $N \cap G_{p}=1$ and obtain the contradiction $N_{G}\left(G_{p}\right) \in g(p)$; therefore $q=p$. Then, since $O_{p^{\prime}}(G)=1$ and $N \subseteq \Phi(G)$, we have $O_{p^{\prime}}\left(N_{G}\left(G_{p}\right) / N\right)=1$, which implies that $O_{p^{\prime}, p}\left(N_{G}\left(G_{p}\right) / N\right)=G_{p} / N$; consequently $G_{p} / N$ is the intersection of the centralisers of all chief $p$-factors of $N_{G}\left(G_{p}\right) / N$.

Now a well-known theorem of Lubeseder (see [4, IV, (4.6) Theorem]) shows that $g(p)$ is locally defined by some formation function $\mathcal{F}$, because, by hypothesis, $g(p)$ is a saturated formation. Then, setting $N_{G}\left(G_{p}\right) / N=\Gamma$, we have $\operatorname{Aut}_{\Gamma}(H / K) \in \mathcal{F}(p)$ for all chief $p$-factors $H / K$ of $\Gamma$, that is $\Gamma / C_{\Gamma}(H / K) \in \mathcal{F}(p)$; it follows $\Gamma / O_{p^{\prime}, p}(\Gamma)$ $=\Gamma / \Gamma_{p} \cong N_{G}\left(G_{p}\right) / G_{p} \in \mathcal{F}(p)$, from which we obtain that Aut $_{N_{G}\left(G_{p}\right)}(H / K) \in \mathcal{F}(p)$, for all chief $p$-factors $H / K$ of $N_{G}\left(G_{p}\right)$ such that $N \subseteq K$. On the other hand, if $H / K$ is a chief $p$-factor of $N_{G}\left(G_{p}\right)$ such that $H \subseteq N$, we have $G_{p} \subseteq C_{N_{G}\left(G_{p}\right)}(H / K)$ and so Aut $_{N_{G}\left(G_{p}\right)}(H / K) \in \mathcal{F}(p)$, because it is a homomorphic image of $N_{G}\left(G_{p}\right) / G_{p} \in \mathcal{F}(p)$; thus Aut $_{N_{G}\left(G_{p}\right)}(H / K) \in \mathcal{F}(p)$, for all chief $p$-factors $H / K$ of $N_{G}\left(G_{p}\right)$. Now, since $N_{G}\left(G_{p}\right) / N \in g(p)=L F(\mathcal{F})$, it is obvious that, for all primes $q$ different from $p$ the group of automorphisms induced by $N_{G}\left(G_{p}\right)$ on a chief $q$-factor belongs to $\mathcal{F}(q)$. Thus we obtain the contradiction $N_{G}\left(G_{p}\right) \in L F(\mathcal{F})=g(p)$.

\section{The SATURATEd FORMATIONS $\mathrm{N} \tilde{f}_{\pi}$}

A function $f: \mathbb{P} \rightarrow$ \{group classes $\}$ is called a [saturated] formation function if $f(p)$ is a [saturated] formation for all $p \in \mathbb{P}$. If $f$ is a formation function, it is well known that the class

$$
L F(f)=\left(G \in \mathcal{S} \mid \operatorname{Aut}_{G}(H / K) \in f(p), \text { for all chief } p \text {-factors } H / K \text { of } G\right) .
$$

is a saturated formation, called locally defined by $f$. Moreover every saturated formation can be locally defined.

If $\pi$ is a subset of the set $\mathbb{P}$ of all primes, the class $\mathcal{S}_{\pi}$ is the class of $\pi$-groups. If $G$ is a group, $G_{\pi}$ denotes a Hall $\pi$-subgroup of $G$; in particular $G_{p}$ is a Sylow $p$-subgroup of $G$ (if $p$ does not divide $|G|, G_{p}=1$ ).

Definition 2.1: Let $f$ be a function $\mathbb{P} \rightarrow$ \{group classes . The function $f^{*}$ is defined as follows

$$
f^{*}(p)=\left(G \in \mathcal{S} \mid \mathrm{N}_{G}\left(G_{p}\right) / G_{p} \in f(p)\right) \quad(p \in \mathbb{P}) .
$$

Lemma 2.2. If $f: \mathbb{P} \rightarrow$ \{group classes $\}$ is a formation function, then $f^{*}$ is a formation function. 
Proof: It is easy to observe that $f^{*}(p)$ is a homomorph. It remains to prove that $f^{*}(p)$ is $\mathrm{R}_{0}$-closed. Let $G$ be a group with two normal subgroups $N_{1}$ and $N_{2}$ such that $G / N_{i} \in f^{*}(p)(i=1,2)$ and $N_{1} \cap N_{2}=1$. If $G_{p} \in \operatorname{Syl}_{p}(G)$ we have

$$
\frac{N_{G}\left(G_{p}\right)}{G_{p}\left(N_{i} \cap N_{G}\left(G_{p}\right)\right)} \cong \frac{N_{G / N_{i}}\left(\left(G / N_{i}\right)_{p}\right)}{\left(G / N_{i}\right)_{p}} \in f(p) \quad(i=1,2) .
$$

It follows, since $f(p)$ is $\mathbf{R}_{0}$-closed, that $N_{G}\left(G_{p}\right) / G_{p} \in f(p)$, observing that

$$
G_{p}\left(N_{1} \cap N_{G}\left(G_{p}\right)\right) \cap G_{p}\left(N_{2} \cap N_{G}\left(G_{p}\right)\right)=G_{p} .
$$

Thus $G \in f^{*}(p)$.

Definitions 2.3: Let $f$ be a formation function and $\pi$ be a (possibly empty) set of primes. The formation function $f / \pi$ is defined as follows:

$$
(f / \pi)(p):=\left\{\begin{array}{ll}
\emptyset & \text { if } p \in \pi \\
f(p) & \text { if } p \notin \pi
\end{array} \quad(p \in \mathbb{P}) .\right.
$$

For every prime $p$ the formation function $(f / \pi, p)$ is defined as follows:

$$
(f / \pi, p)(q):=\left\{\begin{array}{ll}
\emptyset & \text { if } q \in \pi \\
f(p) & \text { if } q=p \notin \pi \\
\mathcal{S} & \text { if } q \neq p \text { and } q \notin \pi
\end{array} \quad(q \in \mathbb{P}) .\right.
$$

The saturated formation $L F((f / \pi, p))$ locally defined by the formation function $(f / \pi, p)$ will be denoted by $\tilde{f}_{\pi}(p)$. Thus two formation functions are defined:

$$
\tilde{f}_{\pi}: p \in \mathbb{P} \rightarrow \tilde{f}_{\pi}(p) \text { and } f_{\pi}^{*}: p \in \mathbb{P} \rightarrow(f / \pi)^{*}(p) .
$$

If $\pi=\emptyset$, we shall use the following notation:

$$
(f, p):=(f / \emptyset, p) \text { and } \tilde{f}:=\tilde{f}_{\emptyset} .
$$

The main result is the following theorem.

THEOREM A. Let $f$ be a formation function and $\pi$ a set of primes. Then:

(i) $\mathrm{N} \tilde{f}_{\pi}$ is a saturated formation;

(ii) $\mathrm{N} \tilde{f}_{\pi}$ is locally defined by the formation function $f_{\pi}^{*}$.

Before we proceed with the proof, let us state some easy consequences of the definitions. 
Proposition 2.4. Let $f$ be a formation function and $\pi$ a set of primes. Then, for every prime $p$, we have

$$
\mathcal{S}_{p^{\prime}} \cap \mathcal{S}_{\pi^{\prime}} \subseteq \tilde{f}_{\pi}(p) \subseteq \mathcal{S}_{\pi^{\prime}}
$$

and therefore $\mathrm{N} \tilde{f}_{\pi} \subseteq \mathcal{S}_{\pi^{\prime}}$. In particular:

(i) $\tilde{f}_{\pi}(p)=\mathcal{S}_{\pi^{\prime}}$, for every $p \in \pi$;

(ii) if $\pi=\emptyset, \mathcal{S}_{p^{\prime}} \subseteq \tilde{f}(p)$ for every prime $p$.

Proof: Let $G \in \tilde{f}_{\pi}(p)=L F((f / \pi, p))$ and let $q$ be a prime dividing $|G|$. If $H / K$ is a chief $q$-factor of $G$, we have $\operatorname{Aut}_{G}(H / K) \neq \emptyset$ and so $(f / \pi, p)(q) \neq \emptyset$; therefore, by definition, $q \notin \pi$. Hence $G$ is a $\pi^{\prime}$-group. Now let $G \in \mathcal{S}_{\mathbf{p}^{\prime}} \cap \mathcal{S}_{\pi^{\prime}}$. If $H / K$ is a chief $q$-factor of $G$, then $q \neq p$ and $q \notin \pi$; therefore, by definition, $(f / \pi, p)(q)=\mathcal{S}$ and so $\operatorname{Aut}_{G}(H / K) \in(f / \pi, p)(q)$. Hence $G \in \tilde{f}_{\pi}(p)$.

The inclusion $\mathrm{N} \tilde{f}_{\pi} \subseteq \mathcal{S}_{\pi^{\prime}}$ is an obvious consequence of the definitions and of the inclusion $\tilde{f}_{\pi}(p) \subseteq \mathcal{S}_{\pi^{\prime}}$.

PROPOSITION 2.5. Let $f$ be a formation function and $\pi$ a set of primes. Then:

$$
f_{\pi}^{*}(p)=\left\{\begin{array}{ll}
\emptyset & \text { if } p \in \pi \\
f^{*}(p) & \text { if } p \notin \pi
\end{array} \quad(p \in \mathbb{P}) .\right.
$$

In particular, if $\pi=\emptyset, f_{\emptyset}^{*}=f^{*}$.

Proof: It follows easily from definitions.

Proposition 2.6. Let $f$ be a formation function and $\pi$ a set of primes. Then, for every prime $p$, we have:

$$
\tilde{f}_{\pi}(p)=\mathcal{S}_{\pi^{\prime}} \cap \tilde{f}(p) .
$$

Proof: It follows easily from the definitions.

PROPOSITION 2.7. Let $f$ be a formation function and $\pi$ a set of primes. Then $L F\left(f_{\pi}^{*}\right)=\mathcal{S}_{\pi^{\prime}} \cap L F\left(f^{*}\right)$.

Proof: Let $G \in L F\left(f_{\pi}^{*}\right)$ and let $p$ be a prime dividing $|G|$. If $H / K$ is a chief $p$-factor of $G$ we have $\operatorname{Aut}_{G}(H / K) \in f_{\pi}^{*}(p)$, that is $G / C \in f_{\pi}^{*}$ where $C=C_{G}(H / K)$. Therefore, by definition of $f_{\pi}^{*}=(f / \pi)^{*}$, we obtain that

$$
N_{G}\left(G_{p}\right) / G_{p}\left(C \cap N_{G}\left(G_{p}\right)\right) \in(f / \pi)(p) ;
$$

it follows $(f / \pi)(p) \neq \emptyset$ and so $p \notin \pi$ and $\operatorname{Aut}_{G}(H / K) \in f^{*}(p)$. Thus

$$
G \in \mathcal{S}_{\pi^{\prime}} \cap L F\left(f^{*}\right) .
$$


The inclusion $\mathcal{S}_{\pi^{\prime}} \cap L F\left(f^{*}\right) \subseteq L F\left(f_{\pi}^{*}\right)$ is an easy consequence of the definitions.

Proposition 2.8. Let $f$ be a formation function and $\pi$ a set of primes. Then:

$$
\mathrm{N} \tilde{f}_{\pi}=\mathcal{S}_{\pi^{\prime}} \cap \mathrm{N} \tilde{f}
$$

Proof: It follows easily from the definitions.

THEOREM 2.9. (A. 1st part.) Let $f$ be a formation function and $\pi$ a set of primes. Then $\mathrm{N} \tilde{f}_{\pi}$ is a saturated formation.

Proof: It follows immediately from Theorem 1.1, by recalling Proposition 2.4. [

THEOREM 2.10. Let $f$ be a formation function. Then $\mathrm{N} \tilde{f}$ is a saturated formation and is locally defined by the formation function $f^{*}$.

Proof: First we prove the inclusion $\mathrm{N} \tilde{f} \subseteq L F\left(f^{*}\right)$. Let $G$ be a group of minimal order in $\mathrm{N} \tilde{f} \backslash L F\left(f^{*}\right)$. Since $L F\left(f^{*}\right)$ is a saturated formation, $G$ belongs to the Q-boundary of $L F\left(f^{*}\right)$ and so $G$ is primitive. Then we have $G=K N$, where $N=\operatorname{Soc}(G)=O_{p}(G)$ (for some prime $p$ ) and $K \in L F\left(f^{*}\right)$. Since $G \notin L F\left(f^{*}\right)$ we have

$$
\operatorname{Aut}_{G}(N) \cong G / N \cong K \notin f^{*}(p),
$$

that is $N_{K}\left(K_{p}\right) / K_{p} \notin f(p)$, where $K_{p}=K \cap G_{p}$. On the other hand, since $G \in \mathrm{N} \tilde{f}$, we have

$$
N_{G}\left(G_{p}\right)=N N_{K}\left(K_{p}\right) \in \tilde{f}(p)=L F((f, p)),
$$

that is $N_{G}\left(G_{p}\right) / C_{N_{G}\left(G_{p}\right)}(A / B) \in f(p)$, for all chief $p$-factors $A / B$ of $N_{G}\left(G_{p}\right)$; it follows that $N_{G}\left(G_{p}\right) / G_{p} \in f(p)$, because $G_{p}=O_{p^{\prime}, p}\left(N_{G}\left(G_{p}\right)\right)$; then, since $N_{G}\left(G_{p}\right) / G_{p}$ $\cong N_{K}\left(K_{p}\right) / K_{p}$, we obtain the contradiction $N_{K}\left(K_{p}\right) / K_{p} \in f(p)$.

Now we are going to prove the inclusion $L F\left(f^{*}\right) \subseteq \mathrm{N} \tilde{f}$.

Let $G$ be a group of minimal order in $L F\left(f^{*}\right) \backslash N \tilde{f}$. Since $N \tilde{f}$ is a saturated formation (Theorem 2.9), $G$ belongs to the Q-boundary of $\mathrm{N} \tilde{f}$ and so is primitive. Then, as above, $G=K N$, where $N=\operatorname{Soc}(G)=O_{p}(G)$ is a minimal normal subgroup of $G$ and $K \in \mathrm{N} \tilde{f}$. Since $G \notin \mathrm{N} \tilde{f}$, there exists a prime $q$ dividing $|G|$ such that $N_{G}\left(G_{q}\right) \notin \tilde{f}(q)=L F((f, q))$. If $q \neq p$, we may suppose $G_{q}=K_{q}\left(G_{q} \subseteq K\right)$ and therefore $N_{G}\left(G_{q}\right)=C_{N}\left(K_{q}\right) N_{K}\left(K_{q}\right) \notin \widetilde{f}(q)$, so there exists a chief $q$-factor $A / B$ of $N_{K}\left(K_{q}\right)$ such that $N_{K}\left(K_{q}\right) / C_{N_{K}\left(K_{q}\right)}(A / B) \notin f(q)$ and this contradicts $K \in \mathrm{N} \tilde{f}$. Thus $q=p$. Therefore we have

$$
N_{G}\left(G_{p}\right)=N N_{K}\left(K_{p}\right) \notin \tilde{f}(p)=L F((f, p))
$$

(where $\left.K_{p}=K \cap G_{p}\right)$ and so $N_{G}\left(G_{p}\right) / O_{p, p^{\prime}}\left(N_{G}\left(G_{p}\right)\right) \notin f(p)$. On the other hand, since $G / N \in \mathrm{N} \tilde{f}$ and $O_{p^{\prime}, p}\left(N_{G}\left(G_{p}\right)\right)=G_{p}$, we get

$$
N_{G}\left(G_{p}\right) / O_{p^{\prime}, p}\left(N_{G}\left(G_{p}\right)\right)=N_{G}\left(G_{p}\right) / G_{p} \in f^{*}(p)
$$


from which we obtain the contradiction $N_{G}\left(G_{p}\right) \in \tilde{f}(p)$.

THEOREM 2.11. (A. 2nd part.) Let $f$ be a formation function and $\pi$ a set of primes. Then $\mathrm{N} \tilde{f}_{\pi}=L F\left(f_{\pi}^{*}\right)$.

Proof: It follows immediately from Theorem 2.10, by recalling Propositions 2.7 and 2.8 .

\section{SOME APPLiCATIONS OF THEOREM A}

In this section, by choosing particular formation functions and set of primes in Theorem A, we obtain some interesting examples of saturated formations, that can be defined by the operation $\mathrm{N}$ and for which therefore it can also be obtained a local definition.

Moreover we obtain, as corollaries, a result of Fedri and Serena on the class $\mathrm{N} U$ [5] and, for soluble groups only, the result of Ballester-Bolinches and Shemetkov cited in the introduction [1].

A class of meaningful examples is obtained if we consider the following well-known saturated formation functions $\widetilde{a}_{n}$ ( $n$ a positive integer), which have been introduced by B. Huppert in [7] (see [8, VI, 8]).

Let $n$ be a positive integer. The formation function $a_{n}$ is defined as follows:

$$
a_{n}: p \in \mathbb{P} \rightarrow \mathcal{A}_{p^{n}-1}
$$

where $\mathcal{A}_{p^{n}-1}$ is the formation of Abelian groups whose exponent divides $p^{n}-1$.

Denote by $\pi(n)$ the set of primes which divide $n$. According to our definitions we have, for every prime $p$ :

$$
\left(a_{n} / \pi(n), p\right): q \in \mathbb{P} \rightarrow \begin{cases}\emptyset & \text { if } q \mid n \\ \mathcal{A}_{p^{n}-1} & \text { if } q=p \nmid n \\ \mathcal{S} & \text { if } q \neq p \text { and } q \nmid n\end{cases}
$$

Let $\tilde{a}_{n}:=\left(\widetilde{a_{n}}\right)_{\pi(n)}$, that is $\tilde{a}_{n}(p)=L F\left(\left(a_{n} / \pi(n), p\right)\right),(p \in \mathbb{P})$. It is well known that

$$
\tilde{a}_{n}(p)=\left(G \in \mathcal{S} \mid(|G|, n)=1 \text { and } \bar{r}_{p}(G) \text { either divides } n \text { or is } 0\right)
$$

where $\bar{r}_{p}(G)$ is the arithmetic $p$-rank of $G$ (see [8, VI, 8.3 Hilfsatz]).

We deduce immediately the following result.

THEOREM B. Let $n$ be a positive integer. Then the class $\mathrm{N} \tilde{a}_{n}$ is a saturated formation and it is locally defined by the formation function

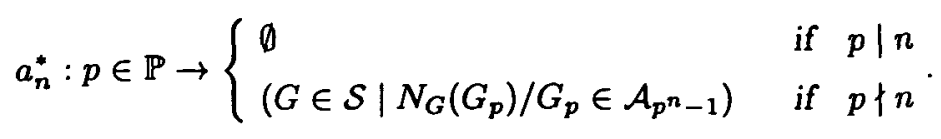


In particular (for $n=1$ ): the class $\mathrm{N} \widetilde{a}_{1}$ of groups, in which normalisers of Sylow $p$ subgroups are $p$-supersoluble, is a saturated formation and is locally defined by the formation function

$$
a_{1}^{*}: p \in \mathbb{P} \rightarrow\left(G \in \mathcal{S} \mid N_{G}\left(G_{p}\right) / G_{p} \in \mathcal{A}_{p-1}\right) .
$$

Theorem. (Fedri-Serena, [5, Proposition 1.2].) Let $p$ and $q$ be primes. Then $\mathcal{S}_{\{p, q\}} \cap \mathrm{N} \mathcal{U}$ is a saturated formation and is locally defined by the formation function

$$
a_{\{p, q\}}^{*}: t \in \mathbb{P} \rightarrow \begin{cases}\emptyset & \text { if } t \neq p, q \\ \left(G \in \mathcal{S} \mid N_{G}\left(G_{p}\right) / G_{p} \in \mathcal{A}_{t-1}\right) & \text { if } t=p \text { or } q .\end{cases}
$$

PROOF: It is enough to observe that $\mathcal{S}_{\{p, q\}} \cap \mathrm{N} \mathcal{U}=\mathcal{S}_{\{p, q\}} \cap \mathrm{N} \tilde{a}_{1}$, therefore the statement follows immediately from Theorem $B$.

The following result is well known.

Proposition 3.3. The (saturated) formation locally defined by the formation function

$$
c: p \in \mathbb{P} \rightarrow\left(G \in \mathcal{S} \mid \operatorname{Syl}_{p}(G)=\operatorname{Carter}(G)\right)
$$

coincides with the formation $\mathcal{N}$ of nilpotent groups.

THEOREM. (Ballester-Bolinches and Shemetkov, [1, Corollary 3].) If the normalisers of Sylow $p$-subgroups of a group $G$ are $p$-nilpotent for every prime $p$, then $G$ is nilpotent.

Proof: Let $e$ be the formation function defined by $e(p)=1$, for all primes $p$. In our notation we have

$$
(e, p)(q)=\left\{\begin{array}{lll}
(1) & \text { if } & q=p \\
\mathcal{S} & \text { if } & q \neq p
\end{array} \quad(q \in \mathbb{P})\right.
$$

and so $\tilde{e}(p)=\mathcal{S}_{p^{\prime}} \mathcal{S}_{p}$ is the formation of $p$-nilpotent groups. Then from Theorem 2.3 we deduce that $N \tilde{e}$ is locally defined by the formation function

$$
e^{*}: p \in \mathbb{P} \rightarrow e^{*}(p)=\left(G \in \mathcal{S} \mid N_{G}\left(G_{p}\right) / G_{p}=1\right)=\left(G \in \mathcal{S} \mid \operatorname{Syl}_{p}(G)=\operatorname{Carter}(G)\right) .
$$

It follows, by Proposition 3.3, that $N \widetilde{e}=\mathcal{N}$ is the formation of nilpotent groups.

\section{REFERENCES}

[1] A. Ballester-Bolinches and L.A. Shemetkov, 'On Normalizers of Sylow Subgroups in Finite Groups', Siberian Math. J. 40 (1999), 1-2. 
[2] M.G. Bianchi, A. Gillio Berta Mauri and P. Hauck, 'On finite groups with nilpotent Sylow normalizers', Arch. Math. 47 (1986), 193-197.

[3] R.A. Bryce, V. Fedri and L. Serena, 'Bounds on the Fitting length of finite soluble groups with supersoluble Sylow normalizers', Bull. Austral. Math. Soc. 44 (1991), 19-31.

[4] K. Doerk and T. Hawkes, Finite soluble groups, de Gruyter Expositions in Maths. 4 (W. de Gruyter, Berlin, 1992).

[5] V. Fedri and L. Serena, 'Finite soluble groups with supersoluble Sylow normalizers', Arch. Math. 50 (1988), 11-18.

[6] G. Glaubermann, 'Prime-power factor groups of finite groups II.', Math. Z. 117 (1970), $46-56$.

[7] B. Huppert, 'Zur Gaschützschen theorie der formationen', Math. Ann. 164 (1966), 133-141.

[8] B. Huppert, Endliche Gruppen I (Springer Verlag, Berlin, New York, 1967).

Dipartimento di Matematica e Applicazioni "R. Caccioppoli"

Università di Napoli "Fderico II"

Complesso Monte S. Angelo, Edificio T, via Cintia

80125 Napoli

Italy

e-mail: daniello@unina.it

clorinda.devivo@dma.unina.it

gabriele.giordano@dma.unina.it 